\title{
Spectroscopic study of the light-induced changes of the core antenna complex CP43 in photosystem II
}

\author{
Y.G. QU, ${ }^{*,+}$ F. ZHOU ${ }^{* *+}$, and T.Y. KUANG ${ }^{* * *}$ \\ College of Life Sciences, Linyi University, 276000 Linyi, China* \\ School of Food Science, Nanjing Xiaozhuang University, 211171 Nanjing, China ${ }^{* *}$ \\ Key Laboratory of Photobiology, Institute of Botany, Chinese Academy of Sciences, 100093 Beijing, China ${ }^{* * *}$
}

\begin{abstract}
In this study, spectroscopic approaches were used to evaluate light-induced changes in chlorophyll protein 43 (CP43). Upon illumination, we observed chlorophyll (Chl) $a$ bleaching with the complete opening of the tetrapyrrole ring. Electronic transition frequencies to different energy levels of the second singlet electronic state $\left(S_{2}\right)$ were changed. In addition, we observed altered rotational and vibrational transitions of Chl $a$ and the CP43 apoprotein as well as variations in the microenvironment surrounding $\mathrm{Chl} a$. CP43 contains three $\beta$-carotene ( $\beta$-Car) and five Chl $a$ molecules, which are involved in the energy transfer from $\beta$-carotene to $\mathrm{Chl} a$. We found that these five $\mathrm{Chl} a$ molecules bleached more readily than others under constant illumination, with energy transfer from $\beta$-Car to $\mathrm{Chl} a$ being continuously lost as illumination continued.
\end{abstract}

Additional key words: absorption; degradation; emission; excitation; fluorescence.

\section{Introduction}

Chl protein 43 (CP43) is encoded by PsbC and is composed of six transmembrane $\alpha$-helical domains as well as five exterior loop domains and stroma exposed N- and C-terminal domains (Bricker and Frankel 2002, Qu et al. 2007). CP43 is located on the surface of PSII core complex, proximal to the $\mathrm{D}_{1}$ of the reaction center (Eijckelhoff et al. 1997, Barber et al. 2000, Hall et al. 2016). This positioning within PSII enables CP43 to readily accept energy absorbed by other membrane proteins such as Chl protein 26 (CP26) and LHCII (Boekema et al. 1999, Yakushevska et al. 2003), and transfer energy to the PSII reaction center wherein water undergoes oxidation and the photochemical reaction occurs (Hughes et al. 2004, Wang et al. 2018).

CP43 contains $4-5 \beta$-carotene ( $\beta$-Car) as well as 13 Chl a molecules in CP43 (Casazza et al. 2010, Müh et al. 2015). Chlorophylls are organized into luminal and stromal layers within the thylakoid membrane, with a Chl molecule (Chl 510) being located centrally within this compartment (Fig. 1).

Light is an important environmental factor that influences the photosynthesis process (Montgomery et al. 2008). When PSII membrane is exposed to an alkaline Tris solution in an aerobic environment, subsequent 5,000 $\mu$ mol(photon) $\mathrm{m}^{-2} \mathrm{~s}^{-1}$ illumination leads to $D_{1}$ and $D_{2}$ degradation. This coincides with the breakdown of CP43 into three fragments $(14,15.5$, and $17.0 \mathrm{kDa}$, respectively) (Yamamoto and Akasaka 1995). Another article reported that CP43 was lost when PSII was treated with light and
Tris. This loss is thought to result from $\mathrm{D}_{1}, \mathrm{Chl}$ protein 47 (CP47), and CP43 cross-linking and a concomitant change in $\mathrm{D}_{1}$ degradation efficiency (Mori et al. 1995).

Prior studies have primarily focused on either studying CP43 in the interior of PSII under alkaline conditions or have studied Chl $a$ molecules in an organic solution. Further studies, specifically focused on individual CP43 complexes, are thus needed in order to better understand its functionality in the absence of other PSII components and under normal $\mathrm{pH}$ value.

In the present study, we employed spectroscopic approaches to evaluate light-induced changes in CP43, with a specific focus on the impairment of $\mathrm{Chl} a$ and changes in energy transfer. Through these approaches, we found that illumination was associated with changes in the frequency of the electronic transition to different energy levels of the second singlet electronic state. This illumination was also associated with altered rotational and vibrational energy transitions for both $\mathrm{Chl} a$ and the CP43 apoprotein, with variations in the $\mathrm{Chl} a$ microenvironment also being evident. A continuous loss of energy transfer from $\beta$-Car to Chl $a$ was also observed in response to illumination, with the five $\mathrm{Chl} a$ molecules in CP43 responsible for accepting energy from $\beta$-Car being bleached more readily than other molecules.

\section{Materials and methods}

CP43 purification: Spinach (Spinacia oleracea L.) PSII particles were isolated using methods previously described

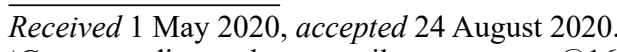

${ }^{+}$Corresponding author; e-mail: yuangangqu@,163.com, zfibcas@163.com

Abbreviations: Chl $a$ - chlorophyll $a$; CP26 - chlorophyll protein 26; CP43 - chlorophyll protein 43; CP47 - chlorophyll protein 47; DM - L-dodecyl maltoside; FWHM - full width at half maximum; $\beta$-Car - $\beta$-carotene.

Acknowledgements: This work was supported by Shandong Provincial Natural Science Foundation, China (ZR2017MC010). 

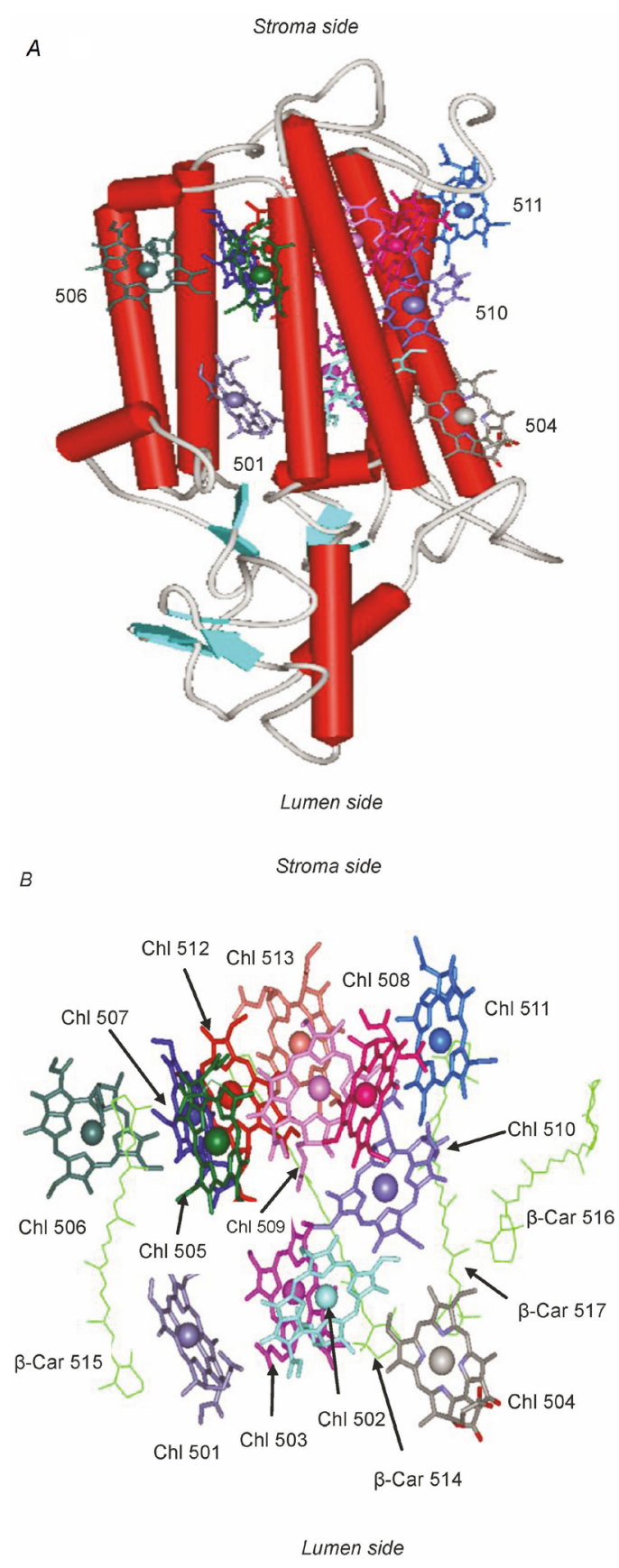

Fig. 1. $\beta$-Car and Chl $a$ organization in CP43 (view along the membrane). (A) Relative positions of Chl $a$ tetrapyrrole rings in CP43 and of the main apoprotein chain of CP43. (B) $\beta$-Car and Chl $a$ organization in CP43. Chls 501-504 are located proximal to the lumenal side of the membrane, Chls 505-513 are to the stromal side. Chl 510 is located within the middle of the membrane. Data were derived from 3JCU in RCSB PDB, and from an article by Wei et al. (2016) pertaining to the spinach (Spinacia oleracea L.) PSII crystal structure. PROTEINVIEW was used to prepare this figure.

by Kuwabara and Murata (1982). Core complex extraction was conducted as previously detailed by Ghanotakis et al. (1989). CP43 purification was conducted as outlined by
Qu et al. (2006) and Picorel et al. (2011). Samples were diluted to $6 \mu \mathrm{g}(\mathrm{Chl}) \mathrm{mL}^{-1}$ in a $0.05 \%$ L-dodecyl maltoside (DM) (Sigma-Aldrich, USA) $20 \mathrm{mmol} \mathrm{L}^{-1}$ Bis-Tris (pH 6.0) solution. All steps were carried out at $4^{\circ} \mathrm{C}$ in the dark.

White-light illumination: A halogen lamp (CDD-1000, Bela Design, China) was used to treat samples with white light at 1,000 $\mu$ mol(photon) $\mathrm{m}^{-2} \mathrm{~s}^{-1}$. CP43 samples were added to a vessel under constant circulation with water at $4^{\circ} \mathrm{C}$, and the sample was continuously stirred during illumination.

Fluorescence measurements: Fluorescence spectra were assessed using an F-4600 fluorescence spectrophotometer (Hitachi, Tokyo, Japan) at room temperature with emission and excitation slits both of $5.0 \mathrm{~nm}$ in size, and with a time constant of $1 \mathrm{~s}$ and a scan rate of $120 \mathrm{~nm} \mathrm{~min}^{-1}$. The detection wavelength was $683 \mathrm{~nm}$ for the fluorescence excitation spectra determination, as it was the peak fluorescence emission spectra position of $\mathrm{CP} 43$.

Absorption spectra measurements: A UH5300 spectrophotometer (Hitachi, Tokyo, Japan) was used to measure room temperature absorption spectra, with a $0.5-\mathrm{nm}$ data interval and a $2 \mathrm{~nm} \mathrm{~s}^{-1}$ scan rate.

Statistical analysis: All experiments were repeated at least three times. The analyses were done with SPSS 20.0 (IBM, Chicago, USA).

\section{Results}

CP43 absorption spectra: The room temperature absorption spectrum of CP43 exhibited two main peaks at 436 and $671 \mathrm{~nm}$ (in the blue and the red region, respectively), with several other small peaks also being evident (Fig. 2). This analysis did not indicate the presence of a pheophytin Qx (1-0) peak at $542 \mathrm{~nm}$, suggesting that the tetrapyrrole ring of Chl $a$ was completely degraded, and did not only loose the $\mathrm{Mg}$ atom. Under illumination, both of these main peaks decreased in size over time, indicating that the $\mathrm{Chl}$ $a$ within CP43 was destroyed over time in an illuminationdependent manner.

No shift in absorption maximum was detected following the sample illumination (Fig. 2, Table 1). The full width at half maximum (FWHM) of the peak at $671 \mathrm{~nm}$ did gradually increase over the first $15 \mathrm{~min}$ of illumination, before rapidly increasing from $15-30 \mathrm{~min}$ and then not changing further after $30 \mathrm{~min}$. The ratio of absorbance at 436 to $419 \mathrm{~nm}$ also decreased in time in response to illumination.

CP43 fluorescence emission spectrum: The fluorescence spectrum is highly sensitive to changes of chromophores in a given protein. We determined that upon excitation at $436 \mathrm{~nm}$ at room temperature, CP43 exhibited a fluorescent emission peak at approximately $683 \mathrm{~nm}$ (Fig. 3). In response to illumination, this fluorescence emission spectrum decreased gradually; with the emission peak 
shifting from $683 \mathrm{~nm}$ to $682.6,682.0,680.8,680.2,679.4$, 678.6 , and $678.0 \mathrm{~nm}$ when illuminated for $2,5,10,15,20$, 30 , and $60 \mathrm{~min}$, respectively.

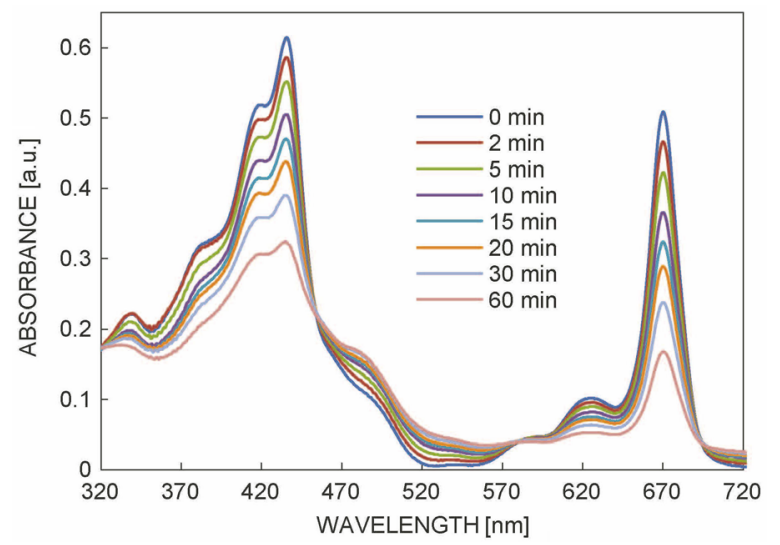

Fig. 2. Changes in the CP43 absorption spectra at room temperature in response to $1,000 \mu \mathrm{mol}$ (photon) $\mathrm{m}^{-2} \mathrm{~s}^{-1}$ illumination. CP43 was suspended in a $0.05 \% \mathrm{DM}, 20 \mathrm{mmol} \mathrm{L}^{-1}$ Bis-Tris, pH 6.0 buffer at a sample density of $6 \mu \mathrm{g} \mathrm{mL}^{-1}$ of Chl $a$.
Upon excitation at $480 \mathrm{~nm}$ at room temperature, $\mathrm{CP} 43$ also exhibited a fluorescent emission peak at approximately $683 \mathrm{~nm}$ (Fig. 4). Following 2, 5, 10, 15, 20, 30, and $60 \mathrm{~min}$ of illumination, these peak values declined by $18.1,35.2$, $51.5,61.2,69.0,75.8$, and $86.4 \%$, respectively, while the peak position shifted from $683 \mathrm{~nm}$ to $682.4,682.0,681.4$, $680.2,679.8,678.8$, and $677.8 \mathrm{~nm}$, respectively.

$\boldsymbol{\beta}$-Car to $\mathrm{Chl} \boldsymbol{a}$ energy transfer rate: In order to calculate the energy transfer rate from $\beta$-Car to Chl $a$, we used the formula F/A $\times 100 \%$ according to the methods of Shan et al. (2000) and de Weerd et al. (2003), where A is corresponding to the absorption intensity at $488 \mathrm{~nm}$ and $\mathrm{F}$ is corresponding to the fluorescence excitation spectrum intensity at $488 \mathrm{~nm}$. We calculated that the energy transfer rate between $\beta$-Car and $\mathrm{Chl} a$ in native $\mathrm{CP} 43$ was $34.1 \%$, which is in line with the previous reports mentioned above. In addition, we found that this energy transfer rate decreased in response to prolonged illumination (Table 2).

The $\beta$-Car and Chl $a$ pairs with energy transfer were presented in Table 3. Data were derived from the spinach (Spinacia oleracea L.) PSII crystal structure (3JCU in RCSB PDB, Wei et al. 2016).

Table 1. Spectrum intensity, FWHM of the peak at $671 \mathrm{~nm}$ and $\mathrm{A}_{436} / \mathrm{A}_{419}$ ratio of CP43 over the course of $1,000 \mu \mathrm{mol}(\mathrm{photon}) \mathrm{m}^{-2} \mathrm{~s}^{-1}$ illumination. Percentage decreased were calculated as $\left(\mathrm{A}_{0}-\mathrm{A}\right) / \mathrm{A}_{0} \times 100 \%$, where $\mathrm{A}$ is the absorbance of CP43 at $671 \mathrm{~nm}$ under illumination, and $\mathrm{A}_{0}$ is the absorbance of CP43 at $671 \mathrm{~nm}$ when without illumination. FWHM - full width at half maximum.

\begin{tabular}{lllllllll}
\hline \multicolumn{7}{l}{ Illumination time $[\mathrm{min}]$} \\
& 0 & 2 & 5 & 10 & 15 & 20 & 30 & 60 \\
\hline Absorbance intensity & 0.539 & 0.496 & 0.452 & 0.395 & 0.353 & 0.319 & 0.267 & 0.198 \\
Percentage decreased [\%] & - & 8.0 & 16.1 & 26.7 & 34.5 & 40.8 & 50.5 & 63.3 \\
FWHM [nm] & 23.8 & 24.2 & 24.3 & 25.3 & 24.8 & 26.5 & 32.0 & 32.0 \\
$\mathrm{~A}_{436} / \mathrm{A}_{419}$ & 1.18 & 1.17 & 1.15 & 1.15 & 1.11 & 1.10 & 1.08 & 1.05 \\
\hline
\end{tabular}

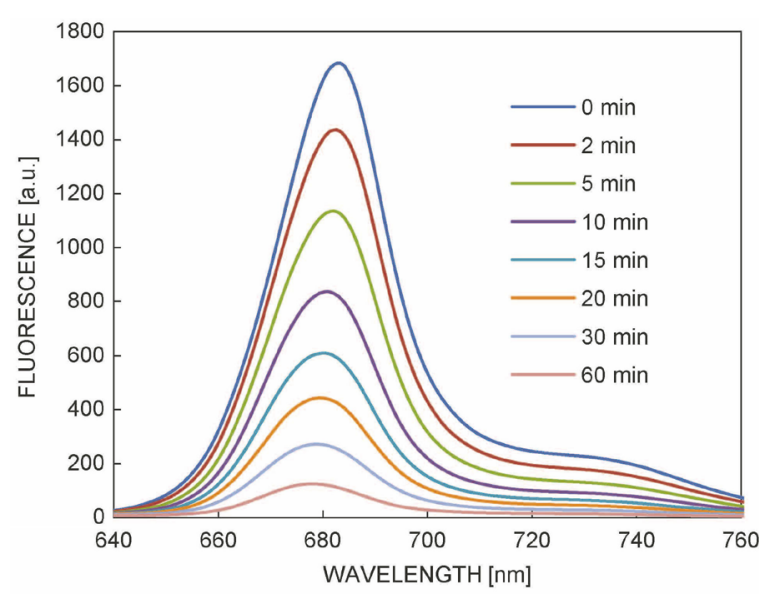

Fig. 3. CP43 fluorescence emission spectra at room temperature following excitation at $436 \mathrm{~nm}$ upon $1,000 \mu \mathrm{mol}$ (photon) $\mathrm{m}^{-2} \mathrm{~s}^{-1}$ illumination. CP43 was suspended in a $0.05 \% \mathrm{DM}, 20 \mathrm{mmol} \mathrm{L}^{-1}$ Bis-Tris, pH 6.0 buffer at a sample density of $6 \mu \mathrm{g} \mathrm{mL}-1$ of Chl $a$.

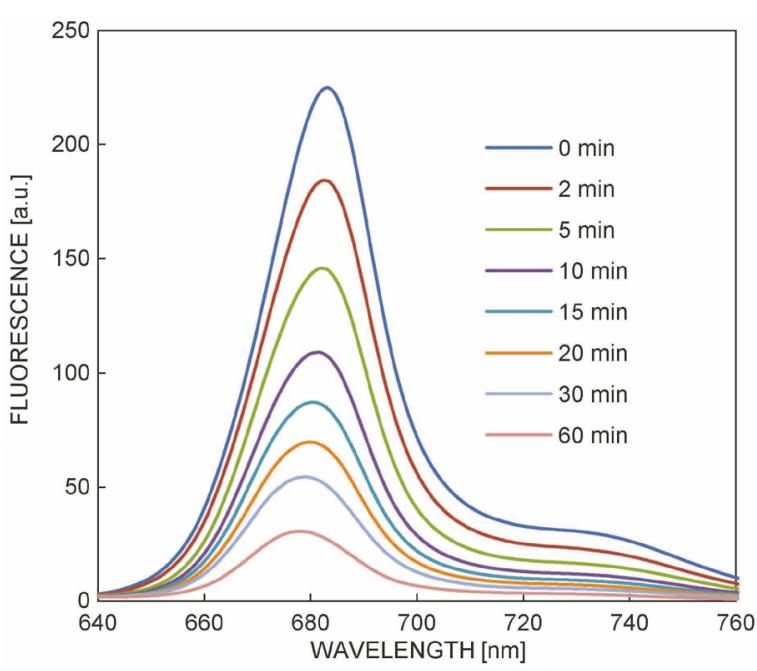

Fig. 4. CP43 fluorescence emission spectra at room temperature following excitation at $480 \mathrm{~nm}$ upon $1,000 \mu \mathrm{mol}$ (photon) $\mathrm{m}^{-2} \mathrm{~s}^{-1}$ illumination. CP43 was suspended in a $0.05 \% \mathrm{DM}, 20 \mathrm{mmol} \mathrm{L}^{-1}$ Bis-Tris, pH 6.0 buffer at a sample density of $6 \mu \mathrm{g} \mathrm{mL}-1$ of Chl $a$. 
Table 2. Energy transfer efficiency from $\beta$-Car to $\mathrm{Chl} a$ in response to $1,000 \mu \mathrm{mol}$ (photon) $\mathrm{m}^{-2} \mathrm{~s}^{-1}$ illumination. Data are mean $\pm \mathrm{SD}$ $(n=3)$. Percentage decreased was calculated as $\left(\mathrm{T}_{0}-\mathrm{T}\right) / \mathrm{T}_{0} \times 100 \%$, where $\mathrm{T}$ is the energy transfer efficiency of CP43 under illumination, and $\mathrm{T}_{0}$ is the energy transfer efficiency of CP43 when without illumination.

\begin{tabular}{|c|c|c|c|c|c|c|c|c|}
\hline & \multicolumn{8}{|c|}{ Illumination time [min] } \\
\hline & 0 & 2 & 5 & 10 & 15 & 20 & 30 & 60 \\
\hline $\begin{array}{l}\text { Energy transfer } \\
\text { efficiency [\%] }\end{array}$ & $34.10 \pm 1.52$ & $27.01 \pm 1.40$ & $19.74 \pm 1.08$ & $13.57 \pm 0.72$ & $9.72 \pm 0.48$ & $6.67 \pm 0.31$ & $3.53 \pm 0.19$ & $0.95 \pm 0.05$ \\
\hline $\begin{array}{l}\text { Percentage } \\
\text { decreased [\%] }\end{array}$ & - & 20.5 & 41.9 & 60.1 & 71.4 & 80.4 & 89.6 & 97.2 \\
\hline
\end{tabular}

Table 3. Distances between $\beta$-Car and Chl $a$ pairs in CP43. Only $\beta$-Car and Chl $a$ pairs with an inter-chromophore distance of $<5 \AA$ are shown. Distances were measured edge-to-edge between the porphyrin of Chl and the $\beta$-Car aryl ring. Data are derived from the spinach (Spinacia oleracea L.) PSII crystal structure (3JCU in RCSB PDB, Wei et al. 2016).

\begin{tabular}{llllll}
\hline & Chl 501 & Chl 505 & Chl 511 & Chl 512 & Chl 513 \\
\hline$\beta-C a r 514$ & - & - & - & $4.59 \AA$ & $3.92 \AA$ \\
$\beta-C a r 515$ & $3.99 \AA$ & $4.7 \AA$ & - & - & - \\
$\beta-C a r 517$ & - & - & $3.85 \AA$ & - & - \\
\hline
\end{tabular}

\section{Discussion}

Electronic transition: The absorption spectra of Chls primarily arise as a consequence of electronic transitions to $S_{1}$ and $S_{2}$ singlet states from the ground state within the tetrapyrrole ring. Ring $\mathrm{V}$ of the tetrapyrrole ring destroys the degeneracy coming from the ring geometry and then splits the two singlet states. Consequently, the resultant absorption spectra of Chl $a$ exhibit two primary bands (B- and Q-bands), with the 419 and $436 \mathrm{~nm}$ peaks being assigned to the B-band (Houssier and Sauer 1970). Microenvironmental factors can alter the electronic transition frequency between different energy levels for a given chromophore, thus altering the associated absorption intensity (Zhao and Zhou 2000). The observed illuminationdependent reduction in the $\mathrm{A}_{436} / \mathrm{A}_{419}$ ratio (Fig. 2, Table 1) indicates a change in the frequency of electronic transition to different energy levels of the second singlet electronic state $\left(\mathrm{S}_{2}\right)$; the $\mathrm{A}_{436} / \mathrm{A}_{419}$ ratio would not change if only bleaching of Chl $a$ happened. This also suggested that there were changes in the microenvironment surrounding Chl $a$ in this experimental context.

Vibrational and rotational energy levels: Electronic, rotational, and vibrational transitions are related to the overall absorption spectra. The electronic transition is associated with the spectrum peak, while the remainder of the spectrum is comprised of a series of vibrational and rotational transitions. As such, the overall appearance of a given absorption spectrum is closely related to the vibrational states of a given chromophore (Albani 2007). The observed illumination-induced increases in FWHM (Fig. 2, Table 1) are consistent with significant changes in the vibrational and rotational transitions of Chl $a$. The electronic states of chromophores are not linked to those of a given protein with which they interact, while the vibrational states of chromophores are influenced by those of the given protein (Renger 1992). As such, changes in Chl $a$ vibrational states are also related to changes in those of the overall CP43 apoprotein.

Microenvironmental polarity surrounding Chl a: Microenvironmental polarity can influence the maximum position of the emission profile of a given chromophore. When the polarity decreases, fluorescence maximum blue shifts (Zhao and Zhou 2000, Xu and Wang 2006). In response to illumination, CP43 exhibited a blue-shifted maximal fluorescence emission profile in response to excitation at $436 \mathrm{~nm}$ (Fig. 3). This suggests a reduction in the microenvironmental polarity surrounding $\mathrm{Chl} a$. Previous works (Groot et al. 1999, Di Donato et al. 2007) suggested a narrow absorption band for CP43 at $5 \mathrm{~K}$, which peaks at $682.5 \mathrm{~nm}$. This band is correlated to a single isolated Chl $a$ molecule, with which other Chl $a$ molecules are weakly coupled. This Chl $a$ is located in a polar environment, and its exact assignment is not clear now. The absorption of this $\mathrm{Chl} a$ originates from specific pigment-protein interactions, and is involved in the polar environment. Namely, the origin of this absorption is environmental rather than excitonic. In addition, this Chl $a$ is lost more easily and it dominates the emission spectrum. Therefore, it contributes more to the blue shift than other Chls.

Energy transfer from $\boldsymbol{\beta}$-Car to $\mathbf{C h l} \boldsymbol{a}$ : Carotenoids are important pigments participating in photosynthetic antenna reactions, wherein they serve as accessory pigments enabling rapid singlet-singlet energy transfer to antenna $\mathrm{Chl}$ and further to the reaction center, thus extending the overall spectral absorption range (Hashimoto et al. 2016, Collini 2019). The shoulder observed at approximately $480 \mathrm{~nm}$ in the CP43 absorption spectrum is attributable 
to $\beta$-Car (Fig. 2), which primarily functions in CP43 via transferring its excitation energy to $\mathrm{Chl} a$. Since $\beta$-Car is essentially nonfluorescent, the observed fluorescence in response to excitation at $480 \mathrm{~nm}$, therefore, reflects such energy transfer from $\beta$-Car to $\mathrm{Chl} a$. Following illumination, fluorescent emission in response to $480 \mathrm{~nm}$ excitation was reduced (Fig. 4). This suggests a sustained loss of energy transfer from $\beta$-Car to Chl $a$, as supported by Table 2 .

Both the fluorescence peak and the overall fluorescence emission spectra of native CP43 upon excitation at $480 \mathrm{~nm}$ was nearly identical to that observed in response to $436 \mathrm{~nm}$ excitation following normalization (Figs. 3, 4). This further supports the model wherein energy transfer between $\beta$-Car and $\mathrm{Chl} a$ occurs, supporting the functional and structural integrity of the CP43 samples used in the present analysis.

The energy transfer from $\beta$-Car to $\mathrm{Chl} a$ occurs through what is known as the 'Dexter mechanism'. In this mechanism context, the small distance $(<5 \AA)$ between two given chromophores is a key determinant of energy transfer (Shan et al. 2000, de Weerd et al. 2002, Qu et al. 2007). There are a total of five Chl $a$ molecules in CP43 that are sufficiently close to $\beta$-Car to allow this mechanism to function (Fig. 1, Table 3). As such, energy transfer from $\beta$-Car to Chl $a$ within CP43 can readily occur between the pigment pairs detailed in Table 3 .

Beside structure stabilization and light harvesting via singlet state energy transfer, carotenoids are very effective quenchers of Chl triplet states, preventing the sensitized formation of singlet state oxygen. In isolated pigmentprotein complexes, the rate of intersystem crossing is significant and the formation of triplet Chls has been shown in vitro, also for CP43 (Carbonera et al. 1992). By prolonged high-light treatment, the normal protective capability of the $\beta$-Car in CP43 becomes overloaded and some singlet oxygen can inevitably escape and damage Chl $a$ (causing bleaching). Carotene is one of the most stable natural pigments. Difference spectra before and after bleaching show that the visible absorption spectra of $\beta$-Car did not change (data not shown). Therefore, the observed reductions in absorption and fluorescence intensity (Figs. 2-4) and in energy transfer from $\beta$-Car to Chl $a$ (Table 2) in this study were primarily the results of Chl $a$ bleaching, including the light bleaching and the singlet oxygen bleaching. In addition, for the Dexter mechanism functioning, the overlap between donor and acceptor electron clouds is required. Any changes in the orientation or distance of $\mathrm{Chl}$ or carotenoids can thus result in a reduction in energy transfer capabilities (Frank and Christensen 1995). Therefore, the decrease in energy transfer from $\beta$-Car to Chl $a$ might thus also be attributable to microenvironmental changes that affected the distance between or orientation of $\beta$-Car and Chl $a$. The details of the microenvironmental changes of CP43 upon illumination remain to be studied further.

The decreases in calculated energy transfer (Table 2) and in fluorescence emission intensity following $480 \mathrm{~nm}$ excitation (Fig. 4) were greater than the observed decreases in absorption intensity (Fig. 2). This suggests that the five Chl $a$ molecules in CP43 capable of accepting energy from $\beta$-Car bleached more readily than did other $\mathrm{Chl} a$ molecules.

\section{References}

Albani J.R.: Absorption spectroscopy theory. - In: Albani J.R. (ed.): Principles and Applications of Fluorescence Spectroscopy. Pp. 1-11. Blackwell Publishing, Ames 2007.

Barber J., Morris E., Büchel C.: Revealing the structure of the photosystem II chlorophyll binding proteins, CP43 and CP47. - BBA-Bioenergetics 1459: 239-247, 2000.

Boekema E.J., van Roon H., Calkoen F. et al.: Multiple types of association of photosystem II and its light-harvesting antenna in partially solubilized photosystem II membranes. Biochemistry-US 38: 2233-2239, 1999.

Bricker T.M., Frankel L.K.: The structure and function of CP47 and CP43 in Photosystem II. - Photosynth. Res. 72: 131-146, 2002.

Carbonera D., Giacometti G., Agostini G. et al:: ODMR of carotenoid and chlorophyll triplets in CP43 and CP47 complexes of spinach. - Chem. Phys. Lett. 194: 275-281, 1992.

Casazza A.P., Szczepaniak M., Müller M.G. et al.: Energy transfer processes in the isolated core antenna complexes CP43 and CP47 of photosystem II. - BBA-Bioenergetics 1797: 1606-1616, 2010.

Collini E.: Carotenoids in photosynthesis: The revenge of the "accessory" pigments. - Chemistry 5: 494-495, 2019.

de Weerd F.L., Dekker J.P., van Grondelle R.: Dynamics of betacarotene-to-chlorophyll singlet energy transfer in the core of photosystem II. - J. Phys. Chem. B 107: 6214-6220, 2003.

de Weerd F.L., van Stokkum I.H.M., van Amerongen H. et al.: Pathways for energy transfer in the core light-harvesting complexes CP43 and CP47 of photosystem II. - Biophys. J. 82: 1586-1597, 2002.

Di Donato M., van Grondelle R., van Stokkum I.H.M., Groot M.L.: Excitation energy transfer in the photosystem II core antenna complex CP43 studied by femtosecond visible/visible and visible/mid-infrared pump probe spectroscopy. - J. Phys. Chem. B 111: 7345-7352, 2007.

Eijckelhoff C., Dekker J.P., Boekema E.J.: Characterization by electron microscopy of dimeric Photosystem II core complexes from spinach with and without CP43. - BBABioenergetics 1321: 10-20, 1997.

Frank H.A., Christensen R.L.: Singlet energy transfer from carotenoids to bacteriochlorophylls. - In: Blankenship R.E., Madigan M.T., Bauer C.E. (ed.): Anoxygenic Photosynthetic Bacteria. Advances in Photosynthesis and Respiration. Pp. 373-384. Springer, Dordrecht 1995.

Ghanotakis D.F., de Paula J.C., Demetriou D.M. et al.: Isolation and characterization of the $47 \mathrm{kDa}$ protein and the $\mathrm{D}_{1}-\mathrm{D}_{2}-$ cytochrome $b$-559 complex. - BBA-Bioenergetics 974: 4453, 1989.

Groot M.L., Frese R.N., de Weerd F.L. et al.: Spectroscopic properties of the CP43 core antenna protein of photosystem II. - Biophys. J. 77: 3328-3340, 1999.

Hall J., Renger T., Picorel R., Krausz E.: Circularly polarized luminescence spectroscopy reveals low-energy excited states and dynamic localization of vibronic transitions in CP43.BBA-Bioenergetics 1857: 115-128, 2016.

Hashimoto H., Uragami C., Cogdell R.J.: Carotenoids and photosynthesis. - In: Stange C. (ed.): Carotenoids in Nature: Biosynthesis, Regulation and Function. Pp. 111-139. Springer, New York 2016.

Houssier C., Sauer K.: Circular dichroism and magnetic circular dichroism of the chlorophyll and protochlorophyll pigments. - 
J. Am. Chem. Soc. 92: 779-791, 1970.

Hughes J.L., Prince B.J., Årsköld S.P. et al.: Photo-conversion of chlorophylls in higher-plant CP43 characterized by persistent spectral hole burning at 1.7K. - J. Lumin. 108: 131-136, 2004.

Kuwabara T., Murata N.: Inactivation of photosynthetic oxygen evolution and concomitant release of three polypeptides in the photosystem II particles of spinach chloroplasts. - Plant Cell Physiol. 23: 533-539, 1982.

Montgomery R.A., Goldstein G., Givnish T.J.: Photoprotection of PSII in Hawaiian lobeliads from diverse light environments. Funct. Plant Biol. 35: 595-605, 2008.

Mori H., Yamashita Y., Akasaka T., Yamamoto Y.: Further characterization of the loss of antenna chlorophyll-binding protein CP43 from Photosystem II during donor-side photoinhibition. - BBA-Bioenergetics 1228: 37-42, 1995.

Müh F., Plöckinger M., Ortmayer H. et al.: The quest for energy traps in the CP43 antenna of photosystem II. - J. Photoch. Photobio. B 152: 286-300, 2015.

Picorel R., Alfonso M., Seibert M.: Isolation and purification of CP43 and CP47 photosystem II proximal antenna complexes from plants. - In: Carpentier R. (ed.): Photosynthesis Research Protocols. Pp. 105-112. Springer, New York 2011.

Qu Y.G., Gong Y.D., Guo S.K. et al.: Structural characteristics of extra-membrane domains and guanidine hydrochlorideinduced denaturation of photosystem 2 core antenna complexes CP43 and CP47. - Photosynthetica 44: 447-453, 2006.

Qu Y.G., Qin.X.C., Wang W.F. et al.: Energy transfer of aromatic amino acids in photosystem 2 core antenna complex CP43 and CP47. - Photosynthetica 45: 266-271, 2007.

Renger G.: Energy transfer and trapping in photosystem II. In: Barber J. (ed.): The Photosystems: Structure, Function and Molecular Biology. Pp. 52-55. Elsevier, London 1992.

Shan J.X., Wang J.S., Li L.B. et al.: The excitation energy transfer between $\beta$-Car and Chl $a$ molecules in PS II core antenna complexes CP43 and CP47. - Chin. Sci. Bull. 45: 1579-1583, 2000.

Wang W., Li X., Wang Q. et al.: The acidic pH-induced structural changes in apo-CP43 by spectral methodologies and molecular dynamics simulations. - J. Mol. Struct. 1152: 177-188, 2018.

Wei X., Su X., Cao P. et al.: Structure of spinach photosystem II-LHCII supercomplex at $3.2 \AA$ resolution. - Nature 534: 69-74, 2016.

Xu J.G., Wang Z.B.: Effects of environmental factors to the fluorescence spectrum and its intensity. - In: Xu J.G., Wang Z.B. (ed.): Fluorescence Analysis Methods. Pp. 49-63. Science Press, Beijing 2006. [In Chinese]

Yakushevska A.E., Keegstra W., Boekema E.J. et al.: The structure of photosystem II in Arabidopsis: localization of the CP26 and CP29 antenna complexes. - Biochemistry-US 42: 608-613, 2003.

Yamamoto Y., Akasaka T.: Degradation of antenna chlorophyllbinding protein CP43 during photoinhibition of photosystem II. - Biochemistry-US 34: 9038-9045, 1995.

Zhao N.M., Zhou H.M.: Study of the microenvironment around a chromophore by absorption. - In: Zhao N.M., Zhou H.M. (ed.): Biophysics. Pp. 282-286. China High Education Press and Springer Heidelberg, Beijing 2000. [In Chinese]

(C) The authors. This is an open access article distributed under the terms of the Creative Commons BY-NC-ND Licence. 